\author{
P. Weybright \\ P. Maly \\ D. Gomez-Hassan \\ C. Blaesing \\ P. C. Sundgren
}

\section{MR spectroscopy in the evaluation of recurrent contrast-enhancing lesions in the posterior fossa after tumor treatment}

Received: 28 October 2003

Accepted: 5 March 2004

Published online: 23 April 2004

(c) Springer-Verlag 2004
P. Weybright $\cdot$ P. Maly

D. Gomez-Hassan $\cdot$ C. Blaesing

P. C. Sundgren ( $\square)$

Department of Radiology,

Neuroradiology Division,

University of Michigan Hospitals,

1500 E. Medical Center Drive, Ann Arbor

MI 48109-0030, USA

E-mail: sundgren@umich.edu

Tel.: + 1-734-7633253

Fax: + 1-734-7642412

\begin{abstract}
Recurrent contrast-enhancing lesions arising within foci of prior brain neoplasms treated with chemotherapy and/or radiation therapy pose a significant diagnostic dilemma, as they may represent recurrent or residual tumor, treatment-related changes, or a combination of both. Those lesions specifically in the posterior fossa are even more difficult to assess, given the technical limitations of 2D CSI in the infratentorial compartment. We explored the feasibility of 2D-CSI MR spectroscopy in the evaluation of recurrent contrastenhancing lesions in eight consecutive patients who had undergone treatment for posterior fossa or brainstem tumors. Mean $\mathrm{Cho} / \mathrm{Cr}$ (choline/creatine) ratios obtained by 2D-CSI in recurrent tumor, treatment-related changes,
\end{abstract}

and normal white matter were 2.93 , 1.62 , and 0.97 , respectively, mean Cho/NAA (choline/N-Acetyl aspartate) ratios were $4.34,1.74$, and 0.93 , and mean NAA/Cr (N-acetyl aspartate/creatine) ratios were 0.74 , 0.92 , and 1.26 , respectively. In conclusion, also in the posterior fossa, MR spectroscopy is likely to be useful as an adjunct to conventional imaging characteristics in distinguishing recurrent tumor from treatment-related changes, irrespectively of the MRS technique used. In most cases spectra of diagnostic quality can be obtained using 2D-CSI to include coverage of both the lesion and its vicinity.

Keywords MRS - 2D-CSI - Brain • Posterior fossa $\cdot$ Tumor $\cdot$ Radiation necrosis

\section{Introduction}

Enhancing lesions that arise on brain MR imaging at the site of a previously treated neoplasm present a significant clinical dilemma. Most of these neoplasms have been subjected to radiation and/or chemotherapy and many do not have specific imaging characteristics that will enable the neuroradiologist to discriminate tumor recurrence from the inflammatory or necrotic change that can result from treatment with radiation and/or chemotherapy. Both typically demonstrate contrast enhancement. It is, therefore, often the clinical course, a brain biopsy, or imaging over a lengthy follow-up interval that enables the distinction of recurrent tumor from a treatment-related lesion and not the specific imaging itself [1]. A non-invasive tool that could differentiate between these entities when a new enhancing lesion is first identified would be invaluable. MR spectroscopy is well suited for this purpose, provided that spectra of diagnostic quality can be obtained.

In the supratentorial compartment, MR spectroscopy (MRS) has proven useful in the evaluation of many central nervous system neoplasms, often enabling the distinction of neoplasm from normal tissue or from radiation necrosis [2]. MRS results have been shown to correlate with histopathology from biopsy [3, 4] and resection specimens [5]. MRS has been used to differentiate between neoplasm and radiation necrosis and has 
shown correlates of radiation necrosis and tumor progression [6]. Also, specific spectroscopic changes that occur after radiation-induced tumor necrosis have been reported [7, 8]. MR spectroscopy has been used with promising results in guiding CNS tumor biopsy and surgical planning [9], and in preoperative grading of gliomas with increased choline content correlating directly with increased tumor grade $[4,10,11]$. The clinical impact of spectroscopy on the assessment of contrast-enhancing lesions that have indeterminate imaging characteristics has also been reported $[12,13]$.

Although the utility of MRS with respect to neoplasm-related lesions has been demonstrated in the supratentorial compartment $[4,10,12-14]$, there are no dedicated reports evaluating the role of MRS in lesions within the infratentorial compartment. Tumors located in the posterior fossa are often histologically unique and are technically challenging with regard to management and treatment. Performing MR spectroscopy in the posterior fossa is difficult because of the close proximity to the skull base, surrounding calvarium, and the relative amount of air- and fluid-filled spaces compared to the brain parenchyma. As a result, characterization of contrast-enhancing lesions in the posterior fossa with MR spectroscopy is technically challenging [15] and has often failed because of bulk magnetic susceptibility artifacts and in-field inhomogeneity.

The feasibility of performing 2D CSI (two-dimensional chemical shift imaging) MR spectroscopy in the posterior fossa on normal brain, using multiple outervolume suppression slices (OVS) for lipid suppression, has been shown [15]. However, in some subjects, extensive artifact occurs and SVS (single voxel spectroscopy) must then be used to generate diagnostic spectra. While SVS generally yields greater signal to noise, it also results in the averaging of metabolite concentrations over a wide region and decreases the sensitivity in detecting focal metabolite differences that may be required to distinguish tumor recurrence from tumor necrosis. Twodimensional-CSI MR spectroscopy enables multiple regions in the posterior fossa to be interrogated during the same acquisition. This enables a sampling of multiple discrete regions, which may be necessary to discern subtle differences between tumor recurrence and necrosis or, perhaps more importantly, the identification of discrete areas of both recurrent tumor and necrosis in the same lesion. In addition, multiple regions of a specific lesion can be explored in an attempt to find the region with the least artifact. However, some lesions may have such severe magnetic field inhomogeneity or be sufficiently large and homogeneous that single-voxel spectroscopy will result in spectra with a quality that is superior to 2D CSI.

As a result, most authors have used SVS in the posterior fossa almost exclusively. SVS has been used to characterize more diffuse disease processes, such as multisystem atrophy [16], spinocerebellar ataxia [17], and alcoholism [18]. To our knowledge, the feasibility and utility of 2D-CSI MR spectroscopy in the evaluation of neoplasms in the posterior fossa and their discrimination from treatment related changes have not been demonstrated.

In this investigation we sought to demonstrate the feasibility of performing 2D-CSI spectroscopy in the evaluation of recurrent contrast-enhancing lesions in the cerebellum and brainstem in eight patients with known neoplasms in these regions who had undergone radiation and/or chemotherapy.

\section{Materials and methods}

Eight patients, including four males and four females (aged 4-61 years, mean 25 years) were evaluated. Their demographic data and tumor histologic types are listed in Table 1. Spectroscopy was performed on these eight consecutive patients who were examined at the request of the referring physician in an attempt to evaluate a recurrent contrast-enhancing lesion as potentially tumor recurrence or tissue necrosis. The duration between the completion of chemotherapy/radiation therapy and the development of a new enhancing lesion on follow-up MR imaging was 8-28 months. Exemption by our Institutional Review Board (IRB) was obtained for this

Table 1 Patient demographics and tumor histologic types

\begin{tabular}{|c|c|c|c|c|c|c|}
\hline Patient no. & Sex & Age & Tumor type & Treatment & Follow-upduration & Type of MRS \\
\hline 1 & M & 61 & Brainstem glioma & Radiation & 8 months & SVS \\
\hline 2 & $\mathrm{~F}$ & 33 & Brainstem glioma & Radiation/chemotherapy & 8 months & 2D-CSI \\
\hline 4 & M & 4 & Medullary glioma & Surgery/chemotherapy & 19 months & 2D-CSI \\
\hline 5 & M & 17 & Medullary glioma & Radiation/chemotherapy & 24 months & 2D-CSI \\
\hline
\end{tabular}


study. Three patients had contrast-enhancing lesions in the brainstem, and in four patients the lesion was in the cerebellum or cerebral peduncles. One patient had lesions in the cerebellum as well as in both cerebral hemispheres.

Examinations were performed on a $1.5 \mathrm{~T}$ scanner (GE Medical Systems, Milwaukee, USA). A conventional MRI examination that was performed in conjunction with the MR spectroscopy included: pre- and postcontrast-enhanced axial and sagittal T1-weighted, axial T2-weighted sequence with fat saturation, axial FLAIR and diffusion-weighted images, and postcontrast-enhanced T1-weighted images in the coronal projection. All MR spectroscopy examinations were performed as the last series in the study and therefore were always performed after the administration of gadolinium (gadopentate).

Seven patients underwent 2D CSI. The following parameters were used for the 2D-CSI spectroscopy: PRESS sequence, TE/TR 144/1000 ms, field of view (FOV) $16 \mathrm{~cm}$, matrix $16 \times 16$, slice thickness $10-20 \mathrm{~mm}$, acquisition 1 average, scan time 4:20 min. The VOI was placed on non-angled contrast-enhanced axial T1weighted or on non-angled FLAIR images, depending on which image best displayed the lesion. The VOI was placed to include both the lesion and adjacent normalappearing cerebellum. Within the VOI, the signal intensity in voxels placed both in the lesion and in adjacent normal-appearing cerebellum was evaluated. During voxel placement by the neuroradiologist, attempts were always made to perform 2D CSI. However, in one patient (no. 7), the 2D CSI produced spectra of insufficient quality and SVS was instead utilized.

Single-voxel spectroscopy was performed in two patients, both with brainstem lesions. The following parameters were used for SVS: PRESS sequence, TE/TR $144 / 1500 \mathrm{~ms}$, FOV $24 \mathrm{~cm}$, matrix $1 \times 1$, scan thickness of $20 \mathrm{~mm}$, scan time $3: 48 \mathrm{~min}$. In one patient (no. 1), the VOI was placed on a non-angled FLAIR image, and in the other patient (no. 7) the VOI was placed on the coronal contrast-enhanced T1-weighted images, which were felt to display the lesion best. In both patients, SVS was performed with the VOI placed over the lesion, and a second examination was performed with the VOI placed in normal appearing occipital white matter.

In addition to the out-of-field-of-view saturation bands that are routinely placed in all MRS examinations, within-field-of-view saturation bands for suppression of osseous regions adjacent to the tissues of interest were placed when necessary, as shown in Fig. 1. Automatic pre-scanning was performed twice before each spectroscopic scan to assure adequate water suppression and acceptable full-width half-maximum (FWHM) values.

The data were postprocessed on a workstation (GE Sun) using Functool 2000. The spectra were analyzed for the signal intensity of the following metabolites: $N$-acetyl-aspartate (NAA), choline (Cho), creatine $(\mathrm{Cr})$ and presence of lactate (Lac). The following ratios were calculated: $\mathrm{NAA} / \mathrm{Cr}$, Cho/Cr, and Cho/NAA. The identity of a contrast enhancing lesion as either tumor recurrence or treatment-related changes was determined based on tissue biopsy in two patients (nos. 6 and 8), and based on subsequent clinical course and imaging followup examinations in the remaining six patients.

\section{Results}

MR spectroscopy performed over the contrast-enhancing lesion identified on diagnostic MRI in the posterior fossa revealed metabolic spectra consistent with tumor recurrence in five of the eight cases, while treatmentrelated changes and lack of tumor was confirmed in three cases.

The imaging features of lesions determined to be tumor recurrence appeared as solitary contrast-enhanc-
Fig. 1a, b Within-field-of-view saturation bands placed in sagittal and coronal projections
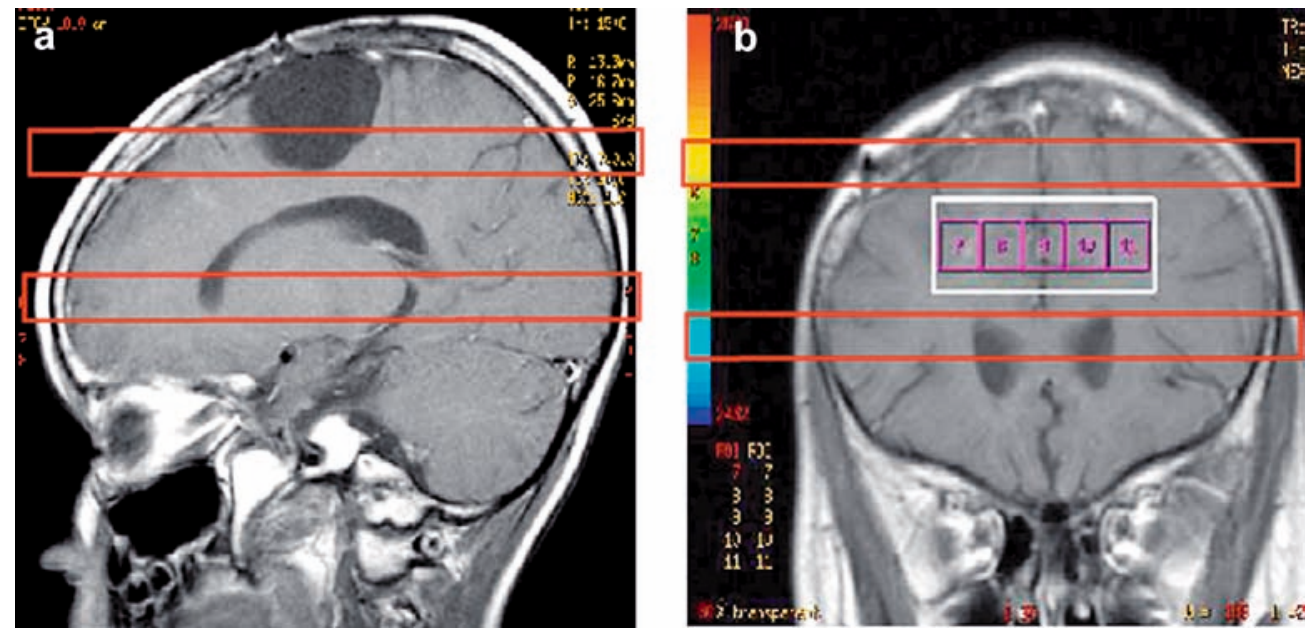

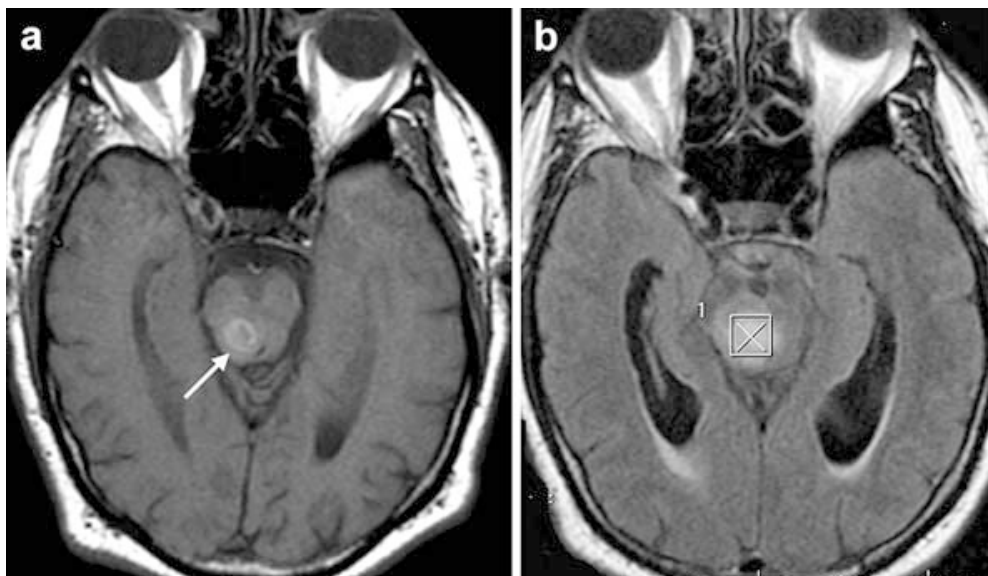

Fig. 2a-c T1-weighted axial images after contrast administration demonstrate a new contrast-enhancing lesion in a 61-year-old male who had undergone radiation therapy for suspected brainstem glioma (a, arrow). SVS (PRESS, TE $144 \mathrm{ms,} \mathrm{TR} 1500 \mathrm{~ms}$ ) was performed owing to the suitable location of the lesion, with the VOI over the contrast-enhancing lesion (b). Significant tenfold elevated signal intensity of the choline and marked decrease of NAA consistent with recurrent tumor (c). Progression on subsequent imaging and of clinical symptoms confirmed the diagnosis of tumor recurrence

ing nodules in the brainstem or cerebellum. These imaging characteristics plus their metabolic ratios were suggestive of tumor recurrence in five patients (patients 1-5) (Figs. 2, 3, 4). This was confirmed by progression of

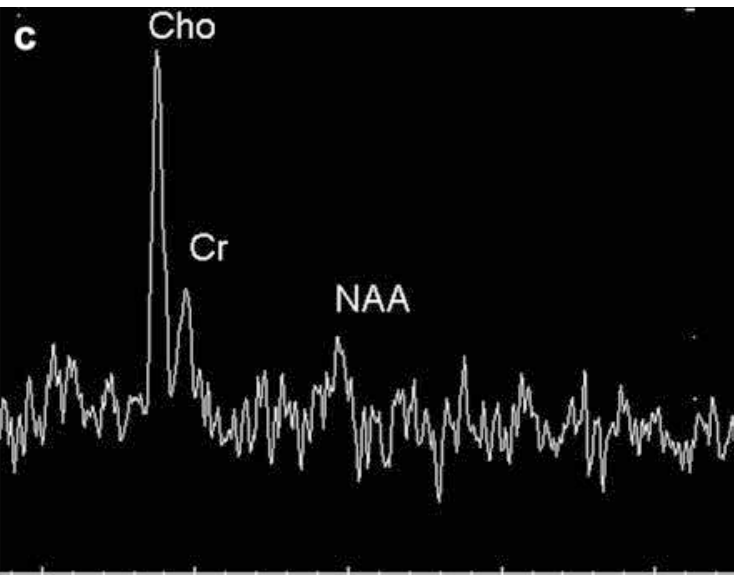

the lesions on subsequent imaging studies and by progression of clinical symptoms. In contrast, in the three cases that showed patchy multiple areas of contrast enhancement, the metabolic spectra were suggestive of

Fig. 3a-c Axial T1-weighted images after contrast administration demonstrate a focal contrast-enhancing lesion in the right cerebellar peduncle in a 33-year-old woman status post resection, chemo- and radiation therapy for a brainstem glioma (a). 2D-CSI (PRESS, TE $144 \mathrm{~ms}$, TR $1000 \mathrm{~ms}$ ) was performed and additional within-field-of-view saturation bands were used to decrease susceptibility artifacts from bone, CSF, and air. The Cho/Cr was markedly elevated (3.04) and the NAA/Cr decreased (0.47). The Cho/NAA ratio was 6.47. The findings were consistent with recurrent neoplasm $(\mathbf{b}, \mathbf{c})$
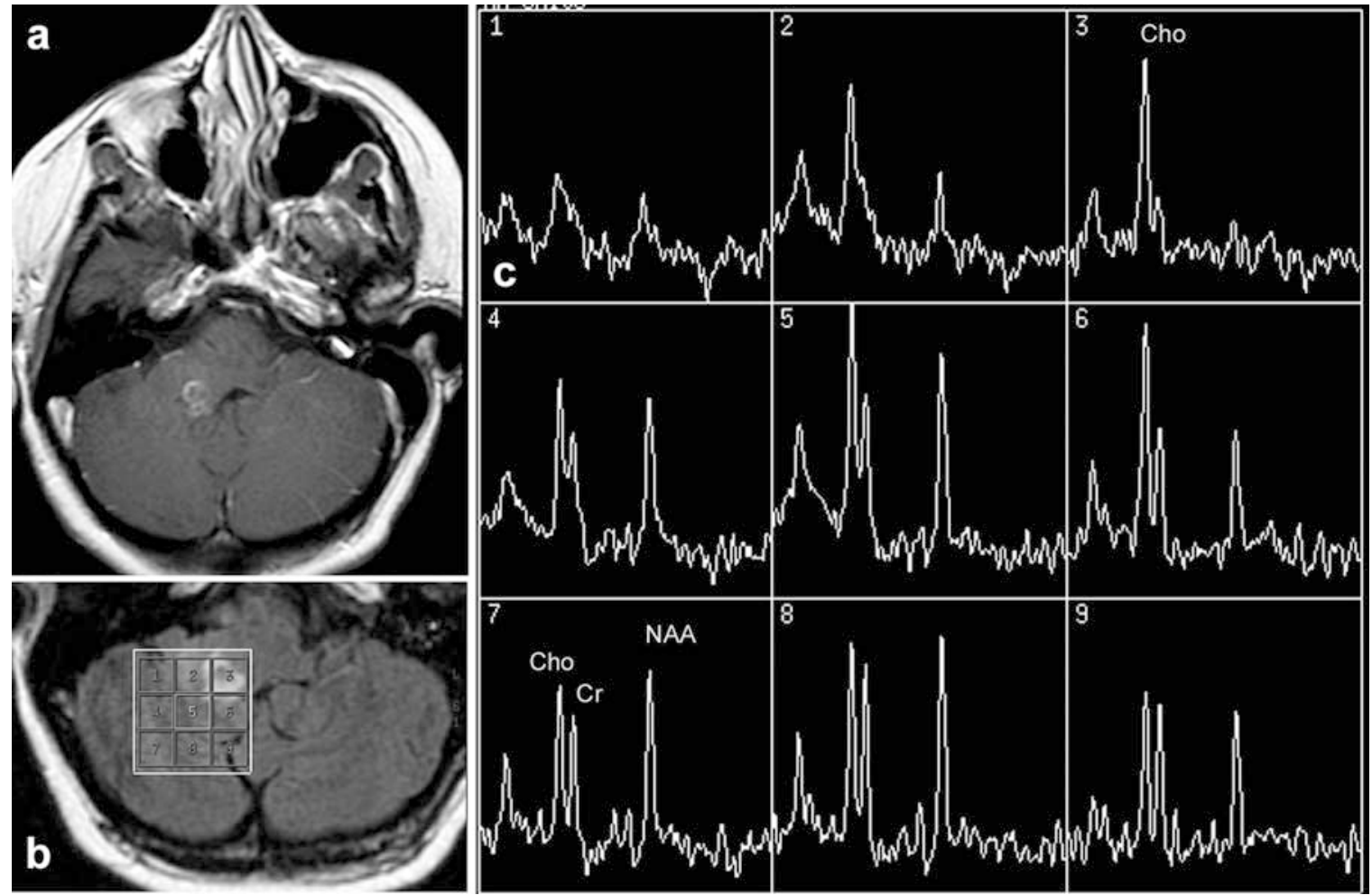



Fig. 4a-c Axial T1-weighted images after contrast administration demonstrate a new contrast-enhancing lesion in a 41-year-old woman who had undergone radiation therapy and surgical resection for a cerebellar astrocytoma 2 years previously (a) (arrows). 2D-CSI (PRESS, TE $144 \mathrm{ms,} \mathrm{TR} 1000 \mathrm{~ms}$ ) was performed with in-field-of-view saturation bands to diminish susceptibility artifacts of CSF, bone and air (b), VOI placed on non-angled axial flair images. The $\mathrm{Cho} / \mathrm{Cr}, \mathrm{NAA} / \mathrm{Cr}$ and $\mathrm{Cho} /$ NAA ratios were $1.65,0.74$ and 2.23 , respectively, and the findings were strongly suggestive of recurrent neoplasm (c). The patient underwent additional chemotherapy for this lesion and has had no progression in the disease to date

treatment-related changes (patients 6-8) (Figs. 5, 6). The absence of tumor was confirmed by biopsy (patients 6 and 8), and by resolution of abnormal contrast enhancement over time (patient 7).

Metabolite ratios for each patient are given in Table 2, and the mean and range of the metabolite ratios in patients with tumor recurrence, in those with treatment-related change, and in normal-appearing white matter are summarized in Table 3. The $\mathrm{Cho} / \mathrm{Cr}$ and Cho/NAA ratios were higher in the tumor lesions (means 2.93, and 4.34, respectively) than in the treatment-related changes (means 1.62, and 1.74, respectively) and were lowest in the normal-appearing white matter (means 0.97 , and 0.93 , respectively). The values obtained by SVS in patients 1 and 7 are not included in these calculations, but displayed separately in Tables 2 and 3. With one exception there was no overlap in the individual $\mathrm{Cho} / \mathrm{Cr}$ and $\mathrm{Cho} / \mathrm{NAA}$ ratios between the three types of tissue. The NAA/Cr ratio was higher in the normal-appearing white matter (mean 1.26) than in the contrast-enhancing areas of tumor (mean 0.74 ) or treatment-related changes (mean 0.92). The presence of a lactate peak was seen in one patient (no.7). While trends are evident, no statistical analysis was performed on these eight patients because of the small sample size.

Single-voxel spectroscopy was performed in two of the eight patients. In the first patient (no. 1) (Fig. 2a-c), only single-voxel spectroscopy was performed, as this lesion was relatively large and homogeneous and its location in the brainstem made it suitable for SVS. Satisfactory spectra were obtained, but the choline was so dramatically increased (greater than tenfold above baseline) that discernible creatine and NAA peaks were not quantifiable.

In the other patient (no.7) (Fig. 6a-d), despite multiple attempts, 2D CSI resulted in suboptimal spectra. SVS was thus performed with voxel placed on coronal images (Fig. 6b, c), resulting in sufficient quality spectra with ratios between the values for normal white matter and those thought to represent recurrence (Table 2), thus suggesting treatment-related changes.

\section{Discussion}

Although the number of patients is small, this study shows that it is possible to perform 2D CSI with highquality spectra in the posterior fossa structures, a technically challenging region, and in a difficult type 


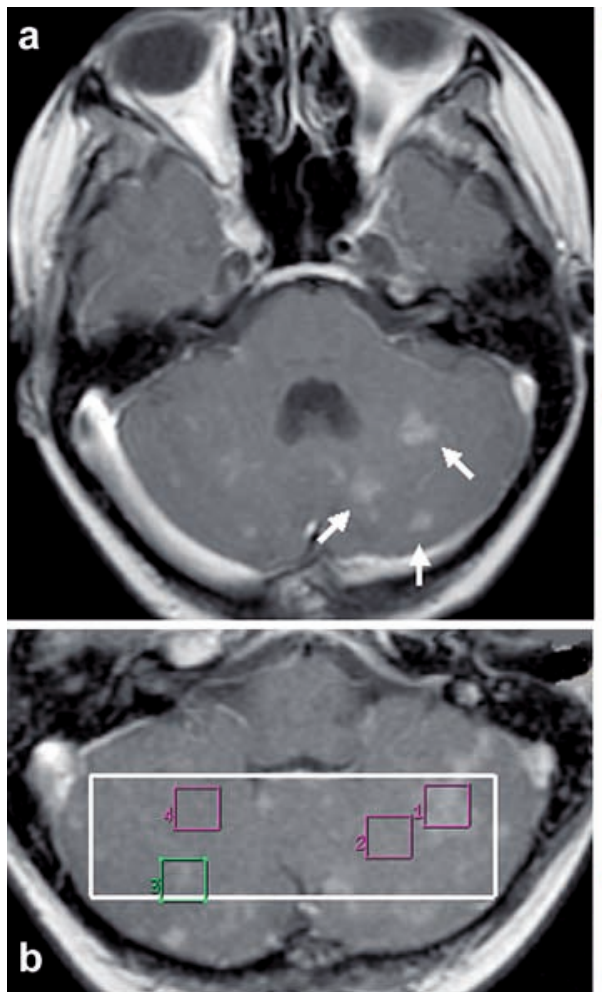

Fig. 5a-c Axial and coronal T1-weighted images after contrast administration demonstrate ill-defined patchy areas of new enhancement involving the vermis and both cerebellar hemispheres (a, arrows). 2D-CSI (PRESS TE/TR 144/1000 ms) was performed with the VOI covering both cerebellar hemispheres (b). Spectra with easily quantifiable choline, creatine, and NAA peaks were obtained. While $\mathrm{Ch} / \mathrm{Cr}$ ratios for this lesion (1.61) were slightly elevated above normal adjacent occipital lobe white matter (0.86), and NAA/Cr ratios were slightly decreased (0.94), neither of these changes was as dramatic as expected for tumor recurrence. The result was interpreted as treatment-related changes (c). Subsequent biopsy of these cerebellar lesions confirmed the presence of tumor necrosis and lack of tumor cells

of lesions in most patients. We have achieved this by optimizing 2D CSI with proper placement of saturation bands, repeat prescanning and using the described imaging parameters, as demonstrated previously [19].

As has been shown in the only similar study examining normal subjects [15], and as in one of our patients (no.7), 2D-CSI may not be of diagnostic quality and information of the metabolic characteristics sometimes must be obtained using SVS. In contrast to the prior study [15] we used in-field-of-view-saturation bands in addition to multiple outer volume suppression slices (OVS) for lipid suppression. These technical considerations helped to reduce various potential artifacts from air, bone, and CSF that are inherently more disturbing in the infra- than the supratentorial compartment.



A range of normal metabolite ratios, depending on the anatomic location within the posterior fossa, has been reported using 2D-CSI (15) and SVS [20]. The $\mathrm{Cho} / \mathrm{Cr}$ ratios for normal white matter in our study (0.83-1.39) are in excellent agreement with $\mathrm{Cho} / \mathrm{Cr}$ ratios in normal white matter $(0.85-1.47)$ reported previously [15], and for NAA/Cr the ratios (1.13-1.52) are also within the realm (1.20-2.33) of those reported by others [15].

A recent analysis of normal volunteers and 43 patients with primary glial tumors has found NAA/Cr ratios significantly lower in the tumor bed after radiation therapy and, moreover, the $\mathrm{NAA} / \mathrm{Cr}$ ratios were inversely proportional to the dose of radiation received [21]. Similarly, in our study, all NAA/Cr ratios in areas of recurrent tumor or treatment-related changes were lower than those in normal white matter. Another study in patients with histopathologically proven supratentorial gliomas treated with radiation therapy has shown lower $\mathrm{Cho} / \mathrm{Cr}$ ratio in those with radiation change than in those with recurrent tumor [22]. In agreement with these results, our results also suggest that the choline content in radiation change lies between that of normal brain tissue and that of recurrent tumor.

The spread of the metabolite ratios is explained in part by differences in the anatomic location $[15,20]$ of each of these lesions, but also by the inevitable volume averaging of normal and abnormal tissues within the same voxel. Single-voxel spectroscopy is particularly prone to such volume averaging, and indeed our 
Fig. 6a-d Coronal T1 weighted images after contrast administration demonstrate focal patchy contrast enhancement in the brainstem and cerebellar hemispheres in a 14 -year-old boy status post resection, chemo- and radiation therapy for a medulloblastoma (a). SVS spectroscopy (PRESS TE/TR $144 / 1500 \mathrm{~ms}$ ) was performed with the VOI placed on coronal images (b). Good-quality spectra were obtained and, although volume averaging of normal tissue and enhancing lesion undoubtedly occurred with the use of single-voxel spectroscopy, the $\mathrm{Cho} / \mathrm{Cr}$, NAA/Cr, and $\mathrm{Cho} / \mathrm{NAA}$ ratios for this lesion were $1.69,0.90$, and 1.88 , respectively. Note was made of inverted lactate peak (c). MRI performed 6 months later demonstrated interval improvement of the abnormalities in the brainstem and cerebellum (d)
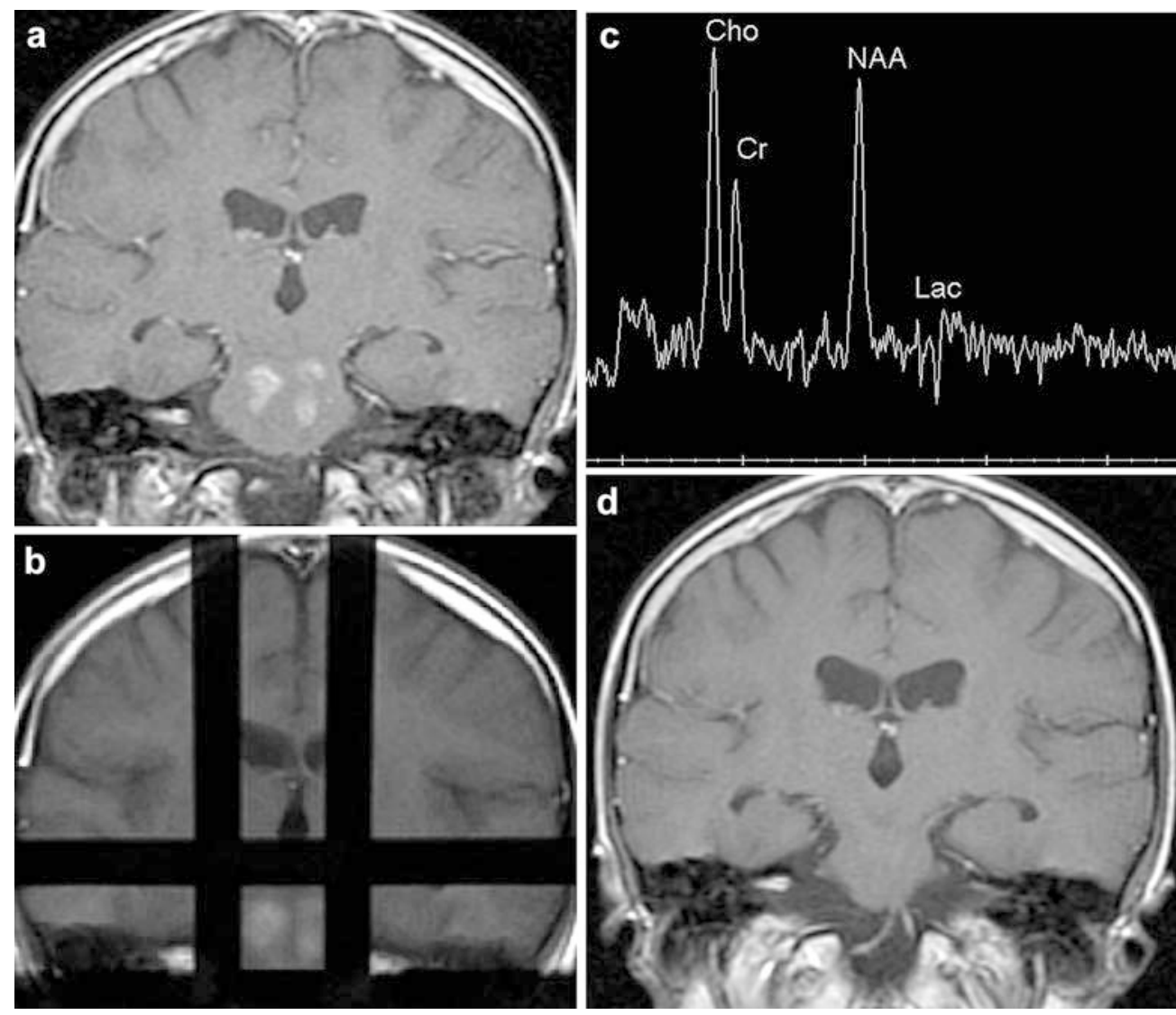

Table 2 The metabolite ratios of the contrast-enhancing lesion in each patient ( $N A$ not available)

${ }^{\mathrm{a}}$ The only overlap among all ratios calculated in this study

Table 3 The metabolite ratios (mean and range) of normal white matter, post-

treatment-related changes, and recurrent tumors

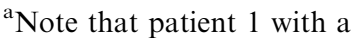
more than tenfold increase of choline is not included in these calculations

\begin{tabular}{lllll}
\hline Patient & $\begin{array}{l}\text { Recurrence or } \\
\text { treatment-related } \\
\text { change }\end{array}$ & Choline/creatine & Choline/NAA & NAA/creatine \\
\hline 1 & Recurrence & Tenfold increase & NA & NA \\
2 & Recurrence & 3.04 & 6.47 & 0.47 \\
3 & Recurrence & 1.65 & 2.23 & 0.74 \\
4 & Recurrence & 2.79 & 2.47 & 1.13 \\
5 & Recurrence & 4.26 & 6.19 & 0.62 \\
6 & Treatment-related change & 1.61 & 1.71 & 0.94 \\
7 & Treatment-related change & $1.69^{*}$ & 1.88 & 0.90 \\
8 & Treatment-related change & 1.64 & 1.78 & 0.92 \\
\hline
\end{tabular}

\begin{tabular}{llll}
\hline Metabolite ratios & $\begin{array}{l}\text { Normal white } \\
\text { matter }(n=6 / 8)\end{array}$ & $\begin{array}{l}\text { Post-treatment } \\
\text { changes }(n=2 / 3)\end{array}$ & $\begin{array}{l}\text { Recurrent } \\
\text { tumor }\left(n=4 / 5^{\mathrm{a}}\right)\end{array}$ \\
\hline Cho/Cr 2D-CSI & $0.97(0.83-1.39)$ & $1.62(1.61-1.64)$ & $2.93(1.65-4.26)$ \\
SVS & 0.96 & 1.69 & $4.34(2.23-6.47)$ \\
Cho/NAA 2D-CSI & $0.93(0.56-1.23)$ & $1.74(1.71-1.78)$ & \\
SVS & 0.46 & 1.88 & $0.74(0.47-1.13)$ \\
NAA/ Cr 2D-CSI & $1.26(1.13-1.52)$ & $0.92(0.87-0.94)$ & \\
SVS & 2.09 & 0.90 & \\
\hline
\end{tabular}

two patients who underwent SVS were at the extreme of the ranges both for the $\mathrm{Cho} / \mathrm{Cr}$ ratio within the lesion and the NAA/Cr ratio in normal white matter.
Specifically in the type of lesions analyzed here, the spread of values is also likely explained by the known scenario in which a recurrent tumor arises in a bed 
of abnormal tissue that is altered by treatment, which results in an inevitable averaging of the metabolite differences that may exist between these two entities. This is supported by findings of authors correlating MRS patterns in supratentorial CNS neoplasms with pathologic results of subsequent tissue analysis who reported that "spectral patterns are less definitive when tissues composed of varying degrees of mixed tumor and necrosis are examined" [3]. However, they found that in the supratentorial neoplasms a $\mathrm{Cho} / \mathrm{Cr}$ ratio greater than 1.79 had a sevenfold increased chance of that tissue being pure tumor [3]. Interestingly, in our study none of the $\mathrm{Cho} / \mathrm{Cr}$ ratios in normal white matter or in treatment-related changes, i.e., not in the recurrent tumor, reached above 1.69 .

In conclusion, also in the posterior fossa, MR spectroscopy is likely to be useful as an adjunct to conventional imaging characteristics in distinguishing recurrent tumor from treatment-related changes, irrespectively of the MRS technique used. In most cases spectra of diagnostic quality can be obtained using 2D-CSI, with coverage including both the lesion and its vicinity.

\section{References}

1. Bonavita S, Di Salle F, Tedeschi G (1999) Proton MRS in neurological disorders. Eur Radiol 30:125-131

2. Fulham MJ, Bizzi A, Dieta MJ, Shih HH, Raman R, Sobering GS, Frank JA, Dwyer AJ, Alger JR, Di Chiro G (1992) Mapping of brain tumor metabolites with proton MR spectroscopic imaging: clinical relevance. Radiology 185:675-686

3. Rock JP, Hearshen D, Scarpace L, Croteau D, Gutierrez J, Fisher JL, Rossenblum ML, Mikkelsen T (2002) Correlations between magnetic resonance spectroscopy and image-guided histopathology, with special attention to radiation necrosis. Neurosurgery 51:912-919

4. Croteau D, Scarpace L, Hearschen D, Gutierrez J, Fischer J, Rock JP, Mikkelsen T (2001) Correlation between magnetic resonance spectroscopy imaging and image-guided biopsies: semiquantitative and qualitative histopathological analyses of patients with untreated glioma. Neurosurgery 49:823-829

5. Dowling C, Bollen AW, Noworolski SM, McDermott MW, Barbaro NM, Day MR, Henry RG, Chang SM, Dillon WP, Nelson SJ, Vigneron DB (2001) Preoperative proton MR spectroscopic imaging of brain tumors: correlation with histopathologic analysis of resection specimens. AJNR Am J Neuroradiol 22:604-612
6. Schlemmer JP, Bachert P, Henze M, Buslei R. Herfarth KK, Debus J, van Kaick G (2002) Differentiation of radiation necrosis from tumor progression using proton magnetic resonance spectroscopy. Neuroradiology 44:216-222

7. Chong VF, Rumpel H, Fan YF, Mukherji SK (2001) Temporal lobe changes following radiation therapy: imaging and proton MR spectroscopic findings. Eur Radiol 11:317-324

8. Chong VF, Rumpel H, Aw YS, Ho GL, Fan YF, Chua EJ (1999) Temporal lobe necrosis following radiation therapy for nasopharyngeal carcinoma: $1 \mathrm{H}$ MR spectroscopic findings. Int $\mathbf{J}$ Radiat Oncol Biol Phys 45:699-705

9. Hall WA, Martin A, Liu H, Truwit CL (2001) Improving diagnostic yield in brain biopsy: coupling spectroscopic targeting with real-time needle placement. J Magn Reson Imaging 13:12-15

10. Nafe R, Herminghaus S, Raab P, Wagner S, Pilatus U, Schneider B, Schon W, Zanella F, Lanfermann H (2003) Preoperative proton-MR spectroscopy of gliomas - correlation with quantitative nuclear morphology in surgical specimen. J Neurooncol 63:233-245

11. Moller-Hartmann W, Herminghaus S, Krings T, Marquardt G, Lanfermann H, Pilatus U, Zanella FE (2002) Clinical application of proton magnetic resonance spectroscopy to the diagnosis of intracranial mass lesions. Neuroradiology 44:371-381

12. Norfray JF, Tomita T, Byrd SE, Ross BD, Berger PA, Miller RS (1999)

Clinical impact of MR spectroscopy when MR imaging is indeterminate in pediatric brain tumors. AJR Am J Roentgenology 173:119-125
13. Lin A, Bluml S, Mamelak AN (1999) Efficacy of proton magnetic resonance spectroscopy in clinical decision making for patients with suspected malignant brain tumors. J Neurooncol 45:69-81

14. Bendszus M, Warmuth-Metz M, Klein R, Burger R, Schichor J, Tonn JC, Solymosi L (2000) MR spectroscopy in gliomatosis cerebri. AJNR Am J Neuroradiol 21:375-380

15. Galanaud D, Le Fur Y, Nicoli F, Denis B, Confort-Gouny S, Ranjeva J-P, Viout P, Pelletier J, Cozzone P (2001) Regional metabolite levels of the normal posterior fossa studied by proton chemical shift imaging. MAGMA 13:127-133

16. Tedeschi G, Litvan I, Bonavita S, Bertolino A, Lundbom N, Patronas NJ, Hallett M (1997) Proton magnetic resonance spectroscopic imaging in progressive supranuclear palsy, Parkinson's disease, and corticobasal degeneration. Brain 120:1541-1552

17. Mascalchi M, Tosetti M, Plasmati R, Bianchi MC, Tessa C, Salvi F (1998) Proton magnetic resonance spectroscopy in an Italian family with spinocerebellar ataxia type I. Ann Neurol 43:244-252

18. Martin PR, Gibbs SJ, Nimmerrichter AA, Riddle WR, Welch LW, Willcott MR (1995) Brain proton magnetic resonance spectroscopy studies in recently abstinent alcoholics. Alcohol Clin Exp Res 19:1078-1082 
19. Blaesing CM, Rohrer SE, GomezHassan D, Maly Sundgren P (2003) Challenging voxel placement for brain MR spectroscopy. SMRT 12th Annual Meeting, Syllabus p 308
20. Mascalchi M, Brugnoli R, Guerrini L, Belli G, Nistri M, Politi LS, Gavazzi C, Lolli F Argenti G, Villari N (2002) Single-voxel long TE 1H-MR spectroscopy of the normal brainstem and cerebellum. J Magn Reson Imaging 16:532-537

21. Rutkoski T, Tarnawski R, Sokol M, Maciejewski B (2003) Proton-MR spectroscopy of normal brain tissue before and after postoperative radiotherapy because of primary brain tumors. Int J Radiat Oncol Biol Phys 56:1381-1389
22. Isobe $\mathrm{T}$, Matsumara $\mathrm{A}$, Anno I, Nagatomo Y, Yoshizawa T, Itai Y (2003) Changes in proton-MRS in glioma patients before and after irradiation and the significance of quantitative analysis of choline-containing compounds. No Shinkei Geka 31:167-172 